\title{
Mean-Entropy-Skewness Fuzzy Portfolio Selection by Credibility Theory Approach
}

\author{
Rupak Bhattacharyya ${ }^{1}$, Mohuya B. Kar ${ }^{2}$, Samarjit Kar ${ }^{1}$, \\ and Dwijesh Dutta Majumder ${ }^{3}$ \\ ${ }^{1}$ Department of Mathematics, National Institute of Technology, Durgapur 713209, India \\ ${ }^{2}$ Department of C.S.E., Heritage Institute of Technology, Kolkata 107, India \\ ${ }^{3}$ Electronics \& Communication Science Unit, Indian Statistical Institute, Kolkata 108, India \\ mathsrup@gmail.com, mohuya_kar@yahoo.com, kar_s_k@yahoo.com, \\ ddmar@hotmail.com
}

\begin{abstract}
In this paper fuzzy mean-entropy-skewness models are proposed for optimal portfolio selection. Entropy is favored as a measure of risk as it is free from dependence on symmetric probability distribution. Credibility theory is applied to evaluate fuzzy mean, skewness and entropy. Hybrid intelligence algorithm is used for simulation. Numerical examples are given in favor of each of the models.
\end{abstract}

Keywords: Fuzzy portfolio selection problem, Credibility theory, Entropy, Skewness, Mean- entropy- skewness model, Hybrid intelligence algorithm.

\section{Introduction}

Different authors like Philppatos and Wilson [1], Philippatos and Gressis [2], Nawrocki and Harding [3], Simonelli [4], Huang [5], Qin et al. [6] used entropy as an alternative measure of risk to replace variance proposed by Markowitz [7]. Entropy is used as risk in the sense that uncertainty causes lose and so investors dislike uncertainty and entropy is a measure of uncertainty. Entropy is more general than variance as an efficient measure of risk because entropy is free from reliance on symmetric probability distributions and can be computed from non-metric data.

Skewness measures the asymmetry of a distribution. Portfolio returns are generally asymmetric and investors would prefer a portfolio return with larger degree of asymmetry when the mean value and risk are same. There will be three goals in portfolio optimization, maximizing the mean and the skewness and minimizing the entropy. Consideration of skewness in portfolio selection was started by Lai [8] and then continued by Konno et al. [9], Chunhachinda et al. [10], Liu et al. [11], Briec et al. [12] etc . All these literatures assume that the security returns are random variables. There are many non-stochastic factors that affect stock markets and they are improper to deal with probability approaches. So Ramaswamy [13], Inuiguichi et al. [14], Li et al. [15] etc. studied fuzzy portfolio selection. But till date none has considered meanentropy- skewness model for portfolio selection problem.

In this paper, in section 2, the definition of fuzzy entropy by Li and Liu [16] is discussed and also credibility theory is used to evaluate the mean and skewness of the 
fuzzy return. In section 3 portfolio selection models are constructed. In section 4 fuzzy simulation integrated genetic algorithm is used to solve the proposed models. In section 5 numerical results are given to illustrate the method followed by conclusions in section 6 .

\section{Mean, Entropy, Skewness by Credibility Theory}

In this section we will discuss credibility theory (c.f. Liu [17]) to have the mean, skewness and entropy of a fuzzy variable.

Definition 1: Suppose that $\xi$ be a fuzzy variable with membership function $\mu_{\xi}$. Then for any set $B \subset \Re$ the credibility of $\xi \in \mathrm{B}$ is defined as:

$$
\operatorname{Cr}\{\xi \in B\}=\frac{1}{2}\left(\sup _{x \in B} \mu_{\xi}(x)+1-\sup _{x \in B^{c}} \mu_{\xi}(x)\right) .
$$

Definition 2: The expected value of $\xi$ is defined as

$$
E[\xi]=\int_{0}^{\infty} \operatorname{Cr}\{\xi \geq r\} d r-\int_{-\infty}^{0} \operatorname{Cr}\{\xi \leq r\} d r .
$$

Definition 3: Suppose that $\xi$ be a fuzzy variable with finite expected value. The skewness of $\xi$ is defined as

$$
S[\xi]=E\left[(\xi-E[\xi])^{3}\right] .
$$

Definition 4: Let $\xi$ be a continuous fuzzy variable and let $T(t)=-t \cdot \ln (t)-(1-t) \cdot \ln (1-$ $t$ ). Then the entropy of $\xi$ is defined by

$$
H[\xi]=\int_{-\infty}^{\infty} T(\operatorname{Cr}\{\xi=r\}) d r .
$$

Definition 5: Let $\xi$ be a discrete fuzzy variable taking values in $\left\{\mathrm{x}_{1}, \mathrm{x}_{2}, \ldots\right\}$ and let $T(t)=-t \cdot \ln (t)-(1-t) \cdot \ln (1-t)$. Then the entropy of $\xi$ is defined by

$$
H[\xi]=\sum_{i=1}^{\infty} T\left(\operatorname{Cr}\left\{\xi=x_{i}\right\}\right)
$$

Example 1: The expected value, skewness and entropy of a triangular fuzzy number $\xi$ $=(\mathrm{a}, \mathrm{b}, \mathrm{c})$ are respectively

$$
E[\xi]=\frac{a+2 b+c}{4}, S[\xi]=\frac{(c-a)^{2}(c-2 b+a)}{32} \text { and } H[\xi]=\frac{c-a}{2} \text {. }
$$

Theorem 1: Let $\tilde{r}_{i}=\left(a_{i}, b_{i}, c_{i}\right)(i=1,2, \ldots, n)$ be independent triangular fuzzy numbers. Then

$$
E\left[\tilde{r}_{1} x_{1}+\tilde{r}_{2} x_{2}+\ldots .+\tilde{r}_{n} x_{n}\right]=\frac{1}{4} \sum_{i=1}^{n}\left(a_{i}+b_{i}+c_{i}\right)
$$




$$
\begin{gathered}
H\left[\tilde{r}_{1} x_{1}+\tilde{r}_{2} x_{2}+\ldots .+\tilde{r}_{n} x_{n}\right]=\frac{1}{2} \sum_{i=1}^{n}\left(c_{i}-a_{i}\right), \\
S\left[\tilde{r}_{1} x_{1}+\tilde{r}_{2} x_{2}+\ldots .+\tilde{r}_{n} x_{n}\right]=\frac{1}{32}\left(\sum_{i=1}^{n}\left(c_{i}-a_{i}\right) x_{i}\right)^{2} \sum_{i=1}^{n}\left(c_{i}+a_{i}-2 b_{i}\right) .
\end{gathered}
$$

Proof: Since $\tilde{r}_{i}=\left(a_{i}, b_{i}, c_{i}\right)$ are triangular fuzzy numbers, by Extension Principle of Zadeh it follows that

$$
\tilde{\mathrm{r}}_{1} \mathrm{x}_{1}+\tilde{\mathrm{r}}_{2} \mathrm{x}_{2}+\ldots .+\tilde{\mathrm{r}}_{\mathrm{n}} \mathrm{x}_{\mathrm{n}}=\left(\sum_{\mathrm{i}=1}^{\mathrm{n}} \mathrm{a}_{\mathrm{i}} \mathrm{x}_{\mathrm{i}}, \sum_{\mathrm{i}=1}^{\mathrm{n}} \mathrm{b}_{\mathrm{i}} \mathrm{x}_{\mathrm{i}}, \sum_{\mathrm{i}=1}^{\mathrm{n}} \mathrm{c}_{\mathrm{i}} \mathrm{x}_{\mathrm{i}}\right),
$$

which is also a triangular fuzzy number. Combining this with the results obtained in example 1 we are with the theorem.

\section{Mean-Entropy-Skewness Model}

Let $\tilde{r}_{i}$ be a fuzzy number representing the return of the $i^{\text {th }}$ security. Let $x_{i}$ be the portion of the total capital invested in security $i, i=1,2, \ldots, n$.

Then $\tilde{r}_{i}=\frac{p_{i}^{\prime}+d_{i}-p_{i}}{p_{i}}$, where $p_{i}$ is the closing price of the $i^{\text {th }}$ security at present, $\mathrm{p}_{\mathrm{i}}^{\prime}$ is the estimated closing price in the next year and $\mathrm{d}_{\mathrm{i}}$ is the estimated dividends in the next year.

Now when minimum expected return $(\alpha)$ and maximum risk $(\gamma)$ are known, the investor will prefer a portfolio with large skewness. It can be modeled as:

$$
\left\{\begin{array}{l}
\operatorname{maximize} S\left[\tilde{r}_{1} x_{1}+\tilde{r}_{2} x_{2}+\ldots .+\tilde{r}_{n} x_{n}\right] \\
\text { subject to } \\
E\left[\tilde{r}_{1} x_{1}+\tilde{r}_{2} x_{2}+\ldots .+\tilde{r}_{n} x_{n}\right] \geq \alpha \\
H\left[\tilde{r}_{1} x_{1}+\tilde{r}_{2} x_{2}+\ldots+\tilde{r}_{n} x_{n}\right] \leq \gamma \\
x_{1}+x_{2}+\ldots .+x_{n}=1 \\
x_{i} \geq 0, i=1,2, \ldots ., n .
\end{array}\right.
$$

When expected return $(\alpha)$ and skewness $(\beta)$ are both not less than some given target values, the investor would aim to minimize the risk; which can be modeled by II.

$$
\left\{\begin{array}{l}
\operatorname{minimize} \mathrm{H}\left[\tilde{\mathrm{r}}_{1} \mathrm{x}_{1}+\tilde{\mathrm{r}}_{2} \mathrm{x}_{2}+\ldots .+\tilde{\mathrm{r}}_{\mathrm{n}} \mathrm{x}_{\mathrm{n}}\right] \\
\text { subject to } \\
\mathrm{E}\left[\tilde{r}_{\mathrm{r}} \mathrm{x}_{1}+\tilde{\mathrm{r}}_{2} \mathrm{x}_{2}+\ldots .+\tilde{\mathrm{r}}_{\mathrm{n}} \mathrm{x}_{\mathrm{n}}\right] \geq \alpha \\
\mathrm{S}\left[\tilde{r}_{1} \mathrm{x}_{1}+\tilde{\mathrm{r}}_{2} \mathrm{x}_{2}+\ldots .+\tilde{\mathrm{r}}_{\mathrm{n}} \mathrm{x}_{\mathrm{n}}\right] \geq \beta \\
\mathrm{x}_{1}+\mathrm{x}_{2}+\ldots .+\mathrm{x}_{\mathrm{n}}=1, \mathrm{x}_{\mathrm{i}} \geq 0, \mathrm{i}=1,2, \ldots ., \mathrm{n} .
\end{array}\right.
$$

When minimum skewness $(\beta)$ and maximum risk $(\gamma)$ is known, the investor would aim to maximize the expected return. It can be modeled as: 


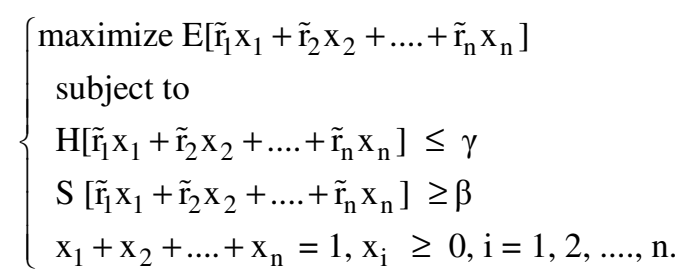

\section{Hybrid Intelligence Algorithm}

To find the optimal portfolio, we integrate fuzzy simulation into the genetic algorithm. The genetic algorithm procedure has been introduced in detail in [18]. Here, we sum up the hybrid intelligent algorithm as follows:

Step 1) In the GA, a solution $\mathrm{x}=\left(\mathrm{x}_{1}, \mathrm{x}_{2}, \ldots, \mathrm{x}_{\mathrm{n}}\right)$ is represented by the chromosome $\mathrm{C}$ $=\left(c_{1}, c_{2}, \ldots, c_{n}\right)$, where the genes $c_{1}, c_{2}, \ldots, c_{n}$ are in the interval $[0,1]$. The matching between the solution and the chromosome is through $x_{i}=c_{i} /\left(c_{1}+c_{2}+\cdots+\right.$ $\left.c_{n}\right), i=1,2, \ldots, n$, which ensures that $x_{1}+x_{2}+\cdots+x_{n}=1$ always holds.

Randomly generate a point $\mathrm{C}$ from the hypercube $[0,1]^{\mathrm{n}}$. Use fuzzy simulation to calculate the entropy value, skewness and expected value. Then check the feasibility of the chromosomes. Take the feasible chromosomes as the initialized chromosomes.

Step 2) Calculate the objective values for all chromosomes by fuzzy simulation. Then, give the rank order of the chromosomes according to the objective values. For modelIII, the greater the expected value is, the better the chromosome is, and the smaller the ordinal number the chromosome has. For model-II, the smaller the entropy value is, the better the chromosome is, and the smaller the ordinal number the chromosome has. Next, compute the values of the rank-based evaluation function of the chromosomes and the fitness of each chromosome according to the rank-based-evaluation function.

Step 3) Select the chromosomes by the roulette wheel selection method, which is fitness proportional.

Step 4) Update the chromosomes by crossover and mutation operations. Check the feasibility of the chromosomes in a similar way as the initial step.

Step 5) Repeat the second to fourth steps for a given number of cycles.

Step 6) Choose the best chromosome as the solution of portfolio selection. For modelIII the chromosome with the maximum expected value is the best chromosome. For model-II the chromosome with the minimum entropy value is the best chromosome. For model-I the chromosome with the maximum skewness is the best chromosome.

\section{Numerical Example}

We apply our mean-entropy-skewness models to the data from Huang [19]. We take the first seven securities. The returns are triangular fuzzy numbers. 


\begin{tabular}{|c|c|c|c|}
\hline Security & $\operatorname{Return}\left(\tilde{\mathrm{r}}_{\mathrm{i}}\right)$ & Security & $\operatorname{Return}\left(\tilde{\mathrm{r}}_{\mathrm{i}}\right)$ \\
\hline I & $(-0.3,1.8,2.3)$ & $\mathrm{V}$ & $(-0.7,2.4,2.7)$ \\
\hline II & $(-0.4,2.0,2.2)$ & VI & $(-0.8,2.5,3.0)$ \\
\hline III & $(-0.5,1.9,2.7)$ & VII & $(-0.6,1.8,3.0)$ \\
\hline IV & $(-0.6,2.2,2.8)$ & \multicolumn{2}{|}{} \\
\hline
\end{tabular}

Example 1. Considering the minimum expected return and the bearable maximum risk to be as 1.6 and 1.8, we judge the model-I and the solution of the above model is obtained as:

\begin{tabular}{|cccccccc|}
\hline \multicolumn{7}{c|}{ Securities } \\
I & II & III & IV & V & VI & VII & Skewness \\
0 & 0 & 0 & 0 & 0 & 0.3077 & 0.6923 & 12.6875 \\
\hline
\end{tabular}

Example 2. Considering the minimum skewness and the bearable maximum risk to be as 12.5 and 1.8, we judge the model-III and the solution of the above model are obtained as:

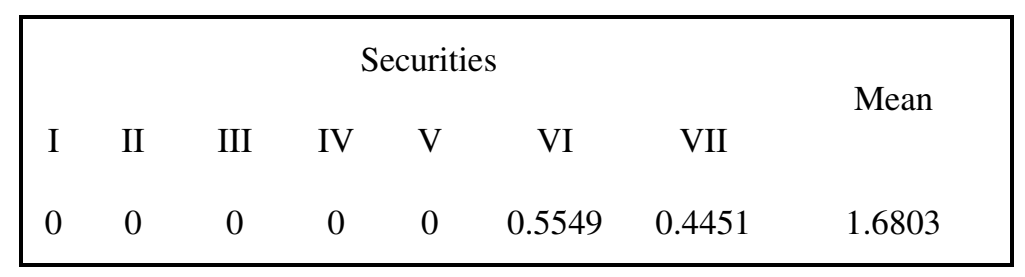

Example 3. : Considering the maximum skewness and the minimum expected return to be as 12.5 and 1.8 , we judge the model-II and the solution of the above model is obtained as:

\begin{tabular}{|cccccccc|}
\hline \multicolumn{7}{c|}{ Securities } & \\
I & II & III & IV & V & VI & VII & Risk \\
0 & 0 & 0 & 0 & 0.0813 & 0.2451 & 0.6736 & 1.772 \\
\hline
\end{tabular}




\section{Conclusion}

Since skewness is incorporated in the portfolio selection model, the model has become more sensible. Entropy is used as the measure of risk. The smaller the entropy value is, the more concentrative the portfolio return is and the safer the investor is. A hybrid intelligence algorithm is designed and numerical results are given to show the effectiveness of the method. Works are going on to apply the proposed approach in Indian stock market.

\section{References}

1. Philippatos, G.C., Wilson, C.J.: Entropy, market risk and selection of efficient portfolios. Applied Economics 4, 209-220 (1972)

2. Philippatos, G.C., Gressis, N.: Conditions of equivalence among E-V, SSD, and E-H portfolio selection criteria: The case for uniform, normal and lognormal distributions. Manag. Sci. 21, 617-625 (1975)

3. Nawrocki, D.N., Harding, W.H.: State-value weighted entropy as a measure of investment risk. Appl. Econ. 18, 411-419 (1986)

4. Simonelli, M.R.: Indeterminacy in portfolio selection. Eur. J. Oper. Res. 163, 170-176 (2005)

5. Huang, X.: Mean-entropy models for fuzzy portfolio selection. IEEE Transactions on Fuzzy Systems 16(4), 1096-1101 (2008)

6. Qin, Z., Li, X., Ji, X.: Portfolio selection based on fuzzy cross-entropy. Journal of Computational and Applied Mathematics 228(1), 188-196 (2009)

7. Markowitz, H.: Portfolio selection. J. Finance 7, 77-91 (1952)

8. Lai, T.: Portfolio selection with skewness: a multiple - objective approach. Review of the Quantitative Finance and Accounting 1, 293-305 (1991)

9. Konno, H., Suzuki, K.: A mean-variance-skewness optimization model. Journal of the Operations Research Society of Japan 38, 137-187 (1995)

10. Chunhachinda, P., Dandapani, P., Hamid, S., Prakash, A.J.: Portfolio selection and skewness: evidence from international stock markets. Journal of Banking and Finance 21, 143 167 (1997)

11. Liu, S.C., Wang, S.Y., Qiu, W.H.: A mean- variance- skewness model for portfolio selection with transaction costs. International Journal of System Science 34, 255-262 (2003)

12. Briec, W., Kerstens, K., Jokung, O.: Mean-variance- skewness portfolio performance gauging: a general shortage function and dual approach. Management Science 53, 135-149 (2007)

13. Ramaswamy, S.: Portfolio selection using fuzzy decision theory. Working paper of Bank for International Settlements 59 (1998)

14. Inuiguchi, M., Ramik, J.: Possibilistic linear programming: a brief review of fuzzy mathematical programming and a comparison with stochastic programming in portfolio selection problem. Fuzzy Sets and Systems 111, 3-28 (2000)

15. Li, X., Qin, Z., Kar, S.: Mean-variance-skewness model for portfolio selection with fuzzy returns. European Journal of Operational Research (2009), doi:10.1016/j.ejor.2009.05.003

16. Li, P., Liu, B.: Entropy of credibility distributions for fuzzy variables. IEEE Transactions for Fuzzy Systems 16(1), 123-129 (2008)

17. Liu, B.: Uncertainty Theory, 3rd edn., http: / / orsc . edu.cn/liu/ut.pdf

18. Huang, X.: Fuzzy chance-constrained portfolio selection. Applied Mathematics and Computation 177, 500-507 (2006)

19. Huang, X.: Mean-semivariance models for fuzzy portfolio selection. Journal of Computational and Applied Mathematics 217, 1-8 (2008) 